\title{
Neuropsychiatric Complexities in the HIV Patient - Clinical Challenges beyond Neurocognitive Disorders
}

Amelia Sim* and Lai Gwen Chan

Institute of Mental Health, Singapore

\begin{abstract}
Objective: To illustrate clinical challenges, explore existing literature and identify knowledge gaps in the management of neuropsychiatric issues beyond neurocognitive disorders in HIV patients.

Methods: Three case vignettes from a consultation-liaison psychiatry setting in a general hospital were collated and summarised into a case series. Clinical questions were identified from each vignette and pertinent literature reviewed to aid a further discussion of management issues.
\end{abstract}

Results: The first case vignette discusses the potential of a multi-dimensional approach to the formulation of a HIV patient with emergent psychiatric and cognitive symptoms. In the second, pharmacological treatment of a HIV patient with a severe manic relapse of Bipolar 1 Disorder and active TB disease is discussed. Lastly, the collective effects of severe Traumatic Brain Injury (TBI) and HIV infection superimposed on that of an ageing brain are described. latrogenic risks of polypharmacy in an elderly HIV patient are further explored.

Conclusion: The practical aspects of clinical challenges regarding the management of HIV patients are multi-fold and most apparent in a real-world setting. Existing literature does not yet adequately address the diagnostic dilemmas and pharmacological complexities faced. The management of other neuropsychiatric manifestations in a HIV patient remains a crucial field to be explored.

Keywords: HIV infection; Neuropsychiatric disorder; HIV psychiatry

\section{Background}

Modern antiretroviral treatments have greatly improved the survival of patients with HIV, resulting in the increasing emergence of a new group of patients that clinicians now have to manage using a chronic disease approach. This group of patients age doubly, both in terms of being at an increased mean age and also as a result of premature ageing from the HIV infection itself, thus placing cognitive problems at the forefront of clinical management $[1,2]$, much like the geriatric patient. Indeed, much literature has been published on milder forms of cognitive impairment aside from HIV-Associated Dementia where there are deficits in cognitive domains such as executive functioning, episodic memory, speed of information processing and motor skills various deficits, culminating in research criteria for the different forms of HIV-Associated Neurocognitive Disorder (HAND) [3] as well as expert consensus guidelines for diagnosis and treatment [4]. General hospital psychiatrists have found that the emphasis for HIV care has shifted to detecting and managing this chronic neurological complication, and rightly so, for there is good evidence that HAND adversely impacts medication adherence, functional outcomes and quality of life [5]. However, an area where existing literature has been inadequate, is the discussion of the real-world clinical challenges of managing an HIV patient with neuropsychiatric complexities including and extending beyond cognitive issues and resulting in difficulties applying the recommended guidelines.

An increased life expectancy also portends the fact that the general hospital psychiatrist needs to manage these patients' pre-existing non-HIV related co-morbidities [6,7]. These can run the gamut from neurological complications of common diseases such as diabetes mellitus and hypertension to neurological or psychiatric illnesses pre-dating the HIV infection. In addition with the passing of time, the HIV patient further accumulates other co-morbidities that add on to a growing list of issues, such as neurodegenerative diseases and neurotrauma.
Challenges such as complex pharmacological interactions, differential diagnoses, interaction of comorbidities and non-classical presentations of common disorders have now become forefront issues psychiatrists have to grapple with, while the management of AIDSrelated symptoms take a momentary backseat. In this paper, we discuss three cases to demonstrate the complex clinical challenges faced in the psychiatric consultation-liaison setting of a general hospital, where co-morbidities such as bipolar disorder, traumatic brain injury and temporal lobe epilepsy interplay with the delicate balance of managing a patient's HIV illness.

\section{Case 1}

The first case is that of a 56 years old Chinese male. He has 3 years of formal education and had been retrenched a year ago as a printing machine operator. Significant medical history included encephalitis in 2002, temporal lobe epilepsy diagnosed in 2007, hypertension and dyslipidemia. There was no past psychiatric history. He was diagnosed with HIV in 2008 after presenting with Pneumocystis Carinii Pneumonia (PCP) and his CD4 count then was $<10$. In 2009, he was treated for Central Nervous System (CNS) Tuberculosis (TB).

In 2014, he presented with cognitive and functional decline over 6 months as well as worsening seizure frequency, increasing from

*Corresponding author: Amelia Sim, Senior Resident, Institute of Mental Health Early Psychosis Intervention Programme, Buangkok Green Medical Park, 10 Buangkok View, Singapore 539747, Singapore; Tel: 65-91820654; E-mail: amelia. sim@mohh.com.sg

Received June 23, 2017; Accepted July 18, 2017; Published July 25, 2017

Citation: Sim A, Chan LG (2017) Neuropsychiatric Complexities in the HIV Patient - Clinical Challenges beyond Neurocognitive Disorders. J AIDS Clin Res 8: 713. doi: 10.4172/2155-6113.1000713

Copyright: (c) $2017 \mathrm{Sim}$ A, et al. This is an open-access article distributed under the terms of the Creative Commons Attribution License, which permits unrestricted use, distribution, and reproduction in any medium, provided the original author and source are credited. 
monthly episodes to fortnightly. Of note, there was marked slowness in recall of information and in verbal and motor responses, resulting in poor work performance and impaired driving ability. There was also increased emotionality where he would cry easily. His antiretroviral (ART) regime consisted of Zidovudine, Lamivudine and Efavirenz which gave a total CNS Penetration Effectiveness (CPE) score of 9 [8]. Symptoms of depression could not be elicited due to marked slowness and reduced verbal output.

On Mental State Examination (MSE), he was a neatly dressed middle-aged Chinese male who was unable to describe his mood and had blunted affect. However, when asked if he felt that life was meaningless, he suddenly burst into tears. There was increased latency of response and poverty of speech. There were no formal thought disorders, perceptual abnormalities or suicidal ideation. On cognitive testing, Mini Metal State Examination (MMSE) score was $12 / 28$ and Montreal Cognitive Assessment (MOCA) score was 15/30, showing deficits of attention, memory and executive functioning. International HIV Dementia Scale was not done as he refused further testing. Neurological examination only showed apraxia on the left. Laboratory testing showed a CD4 count of 477 and undetectable HIV viral load. Magnetic Resonance Imaging (MRI) of the brain showed bilateral chronic lacunar infarcts and microhaemorrhages, small vessel disease, asymmetric right hippocampal atrophy and mild diffuse involutional changes. Lumbar puncture for cerebrospinal fluid viral load measurement was unsuccessful despite fluoroscopic guidance.

He was started on Memantine by his neurologist but had to stop due to worsening seizure frequency. Interestingly, a therapeutic trial of Escitalopram for Depression resulted in not only improvements in terms of emotional dyscontrol, but also with seizure frequency and overall functioning. However there were no improvements in cognitive scores.

This case illustrates the challenges with differential diagnoses in a complex neuro-psychiatric presentation of a HIV patient. The differential diagnoses considered in this case were that of Depressive Disorder, Vascular Depression, Alzheimer's Dementia, Vascular Dementia and HAND. His risk factors for HAND were his low levels of education, low nadir CD4, history of an opportunistic infection in the CNS and presence of possibly neuro-toxic medications [9]. Depressive disorders [10], vascular lesions in the brain and vascular risk factors [11] are all highly prevalent in the HIV population. Accelerated aging in HIV also raises the possibility of neurodegenerative conditions like Alzheimer's dementia [12] especially since hippocampal atrophy and cerebral involution was present. The MRI findings may also indicate an element of emotional lability after stroke [13], in addition to asymmetrical hippocampal atrophy possibly from the effects of encephalitis and epilepsy. His clinical status also made full neuropsychological testing for diagnostic clarification unfeasible. As demonstrated in this case, there exists patients for whom guidelines cannot be applied and an alternative diagnostic approach is necessary. Consideration of a multi-modal picture such as new-onset psychiatric and/or age-related co-morbidities superimposed on that of pre-existing neurological disease accounts for the possibility that the presentation of HIV-associated diseases can be confounded. In addition, a diagnostic trial of antidepressants can be helpful to differentiate pseudo-dementia from dementia and de-lineate the extent of dual diagnoses.

\section{Case 2}

The second case is that of a 41 year old Chinese male with a background history of Bipolar I Disorder. He had previously required Electroconvulsive Therapy (ECT) for mania, but was now stable on a maintenance dose of Quetiapine $400 \mathrm{mg}$ nocte and Valproic Acid 500 mg twice daily. He was diagnosed with HIV in 2015 after presenting with disseminated $\mathrm{TB}$ and a likely tuberculous lesion in the right cerebellum. At time of diagnosis, his CD4 count was $<20$ and viral load 246,300 copies. He was started on high-dose steroids to reduce the mass effect of the cerebellar lesion and also TB treatment with Rifampicin, Isoniazid, Ethambutol and Pyrazinamide. In addition, he required Clarithromycin prophylaxis for atypical mycobacterial infection. His ART regime included Tenofovir, Lamivudine and Ritonavir-boosted Atazanavir which gave a total CPE score of 5 . In the course of his hospitalization however, he suffered a manic relapse and bit a nurse in his agitated state, requiring physical and chemical restraints for himself and necessitating post-exposure HIV prophylaxis for the victim.

On MSE, he was a middle-aged Chinese male of small build. He was restless and animated, with elated mood and affect. He had a grandiose delusion of possessing healing powers with martial arts and of being able to heal himself of HIV. He denied hallucinations in any sensory modality. Pressured speech and flight of ideas were observed. There were no focal neurological signs on physical examination.

This case illustrates the differential diagnoses of mania in a HIVpositive patient and the pharmacological considerations in the acute management of a patient who is experiencing relapses of his multiple diagnoses. Given the advanced state of HIV at the point of diagnosis, the phenomenon of AIDS mania [14,15] is certainly a diagnostic consideration, with the cerebellar lesion and exposure to a potent steroid as contributing organic factors. However, given his pre-existing history of Bipolar Disorder I, it is also likely that his manic relapse had been precipitated by stress from his physical illnesses and the prolonged hospitalization.

Perhaps more importantly, reduced plasma levels of his maintenance psychotropics may have contributed to his relapse due to drug-drug interactions with Rifampicin and Clarithromycin, both having potent effects on hepatic enzymes [16,17].

Furthermore, pharmacological considerations for treatment of Bipolar Disorder in the HIV patient span across not only the acute phase of mania but also during long-term maintenance therapy. TB medications, ARTs and other additional medications, in this case, Clarithromycin, have to be checked for drug-drug interactions with acute phase and maintenance psychotropics. At the same time, it is essential to keep in mind that treatment for TB and prophylaxis for atypical mycobacterium are both time-limited treatments that will be stopped at defined points in the course of illness. When the courses of antibiotics have been completed, the doses of both psychotropics as well as ARTs will have to be decreased in order to avoid drug toxicity. Hence, the clinician's choice of ARTs will have to be carefully considered, especially since the patient is still relatively ART-naïve at this point. There is a role here for the psychiatrist to collaborate with the HIV physician and advocate for the patient's mental health care needs. ECT is an option for a manic patient who does not respond adequately to psychotropics, but for this patient, the presence of the cerebellar lesion may be a contraindication for ECT [18]. In view of the potential risks of harm to self and others during a manic relapse, combination therapy of psychotropics or doses exceeding the maximum recommended dose may also have to be used with the appropriate medical monitoring [19].

\section{Case 3}

The third case is that of a 64 year old Chinese male, unemployed and not formally educated, with a background medical history of 


\begin{tabular}{|c|c|}
\hline Challenges & Recommendations \\
\hline Diagnostic Challenges & Diagnostic trial of psychotropics \\
\hline \multirow{3}{*}{$\begin{array}{ll}\text { - } & \text { Lack of accurate psychiatric history due to patients' poor insight } \\
\text { - } & \text { Incomplete neuropsychological testing due to poor clinical status } \\
\text { - } & \text { Lack of spinal fluid analysis } \\
\text { - } & \text { Confounders of pre-existing disease on top of new-onset disease }\end{array}$} & Formulation of diagnoses considering multiple aetiologies \\
\hline & Use clinician-rated scales as opposed to patient-rated scales \\
\hline & Longitudinal observation and regular reviews of diagnoses at appropriate intervals \\
\hline \multirow{6}{*}{ 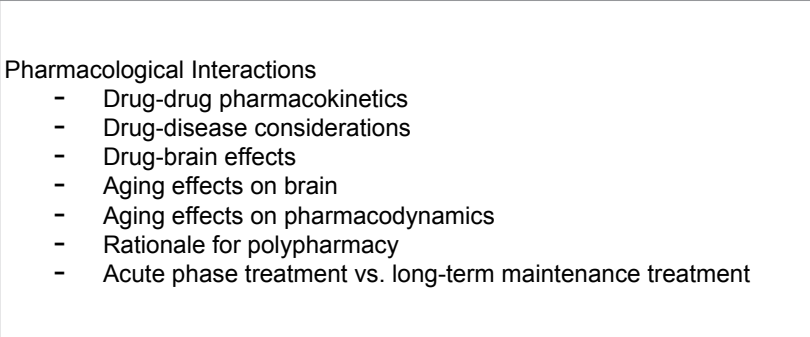 } & $\begin{array}{l}\text { Consider interactions with possible need for maintenance psychotropics when deciding } \\
\text { on initial choice of anti-retroviral therapy }\end{array}$ \\
\hline & $\begin{array}{l}\text { Anticipating psychotropic changes when moving between acute and maintenance } \\
\text { phases of psychiatric illness }\end{array}$ \\
\hline & $\begin{array}{l}\text { Setting time points for reassessment of pharmacological regime at turning points of } \\
\text { illness }\end{array}$ \\
\hline & $\begin{array}{l}\text { Watch for metabolic and cardiovascular side-effects of psychotropics especially in the } \\
\text { older person }\end{array}$ \\
\hline & Be mindful of cognitive reserve and its impact on illness and drug effects \\
\hline & Maintain close collaboration of HIV physician with psychiatrist \\
\hline
\end{tabular}

Table 1: Recommendations for overcoming challenges in the management of psychiatric comorbidities in HIV [32-39].

hypertension and old strokes. At time of HIV diagnosis, his CD4 count was 131 and viral load was 164 copies. This had improved to a CD4 count of 176 and undetectable viral load after 3 months of ART consisting of Abacavir, Lamivudine and Efavirenz. 6 months after diagnosis, he was involved in a road traffic accident as a helmetless motorcyclist and suffered a severe Traumatic Brain Injury (TBI) and skull fractures. Glasgow Coma Scale (GCS) was 7 at the accident scene and 9 on arrival at the Emergency Department. Computed Tomography (CT) of the head showed left subdural haemorrhage, right extradural haemorrhage, pneumocephalus from skull and facial fractures and diffuse axonal injury. $\mathrm{He}$ also had several other musculoskeletal injuries and was managed in the neurosurgical intensive care unit with intracranial pressure monitoring and intravenous mannitol.

Upon transfer to the inpatient neurorehabilitation unit, he remained in post-traumatic amnesia [20] and continued to have severe sleepwake disturbance and bouts of agitation. In the day he required one-toone nursing care and constant distraction through engagement with a variety of activities. There were episodes of severe aggression resulting in damage to objects and furniture. High-doses of Olanzapine were used for tranquilization in addition to Lorazepam and Zopiclone for night sedation, but to no avail. Furthermore, antiretroviral treatment was changed from Efavirenz to Ritonavir-boosted Atazanavir to reduce possible neurotoxicity $[21,22]$. His convalescence was unfortunately complicated by a fall with further head injury as well as a myocardial infarction.

MSE showed an elderly Chinese male who was disoriented to time, place and person. He was inattentive and constantly asking to go home or for his son to come. He was restless and when left alone, would struggle against his restraints and shout for attention. Abnormalities of mood and perception could not be elicited. In the wake of his fall and cardiovascular event, all antipsychotics and sedatives were temporarily stopped to minimise the risks of further complications [23,24]. As he progressed, psychotropics (Olanzapine, Melatonin Prolonged Release and Methylphenidate) were gradually reintroduced at low doses to help regulate his sleep-wake cycle and agitation. He was eventually discharged to a nursing home and as of the latest outpatient review, it was reported that his bouts of agitation and sleep-wake disturbance had settled. Psychotropics were consequently down-titrated with the eventual intention for tailing off.

This case is used to discuss the combined effects of TBI and HIV on the brain of an elderly person, as well as unique pharmacological considerations. There has been one study so far that showed that a TBI increases the risk of neurocognitive impairment in HIV [25]. This study has not been replicated. This patient has risk factors for HAND (advanced age, low educational attainment, low nadir CD4) [9]. His cognitive reserve is also likely to have been reduced since he would be regarded as an "elderly" patient due to the effects of accelerated aging in HIV [1,2]. The risk associated with the use of atypical antipsychotics for behavioural difficulties in the elderly with dementia has been well-documented and should be extrapolated to this case as well [26]. Perhaps, the slightly older HIV patient is equally vulnerable to cardiovascular morbidity and mortality as the geriatric patient. Similarly, the risks of falls associated with the use of benzodiazepines in the elderly should also be a concern [27]. Furthermore, the use of benzodiazepines has the potential to hinder cognitive recovery in this patient [28]. However, pharmacological options for regulating sleepwake disturbances are, in reality, limited. Nevertheless, this was one of the more important treatment goals for him as it caused extreme difficulties in nursing care. The potential neurotoxicity from Efavirenz should also be considered in this patient especially since his cognitive reserve is severely reduced [21,22].

\section{Discussion and Conclusion}

A literature search on the neuropsychiatry of HIV reveals an emphasis on the diagnosis and management of neurocognitive impairment in HIV [29]. Full neuropsychological testing is the recommended standard for diagnosis while considering psychiatric differential diagnoses concurrently $[3,4]$. However, existing literature does not adequately address the practical challenges and complexities in clinical practice where many patients are unable to undergo neuropsychological testing. Most studies were conducted among stable outpatients, thus missing out on the most complex patients who are usually in the inpatient setting. It can be seen from these 3 cases that the presence of comorbid issues and atypical presentations necessitate detailed and comprehensive neurological assessments and psychiatric formulations. The use of screening tools such as mood and anxiety questionnaires as recommended in expert consensus guidelines would have been insufficient. These cases also highlight the fact that there are instances when HAND cannot be easily or confidently diagnosed.

Furthermore, literature on the pharmacological complexities in the treatment of medical and psychiatric comorbidities of HIV patients remains a relatively unreported field. As can be seen in the cases discussed, the general hospital psychiatrist needs to account for the complex interplay of drug-drug, drug-disease, drug-brain and diseasebrain interactions. Table 1 illustrates several recommendations for overcoming this diagnostic and pharmacological challenges. 
Citation: Sim A, Chan LG (2017) Neuropsychiatric Complexities in the HIV Patient - Clinical Challenges beyond Neurocognitive Disorders. J AIDS Clin Res 8: 713. doi: 10.4172/2155-6113.1000713

Page 4 of 4

As aging with HIV becomes commonplace, it is inevitable that psychiatrists will be facing more of such complex patients to manage especially since available studies have reported a higher prevalence rate of psychiatric and medical co-morbidities in HIV patients [30,31]. Specifically-trained HIV psychiatrists are few in number, so the onus is on the general hospital psychiatrist to combine up-to-date knowledge with clinical acumen and sound clinical practice so as to provide these complex patients with the best available care.

\section{References}

1. Capeau J (2011) Premature aging and premature age-related comorbidities in HIV-infected patients: Facts and hypotheses. Clin Infect Dis 53: 1127-1129.

2. Deeks Steven G, Andrew N Phillips (2009) Clinical review: HIV infection, antiretroviral treatment, ageing and non-AIDS related morbidity. BMJ 338: 288292

3. Antinori A, Arendt G, Becker JT, Brew BJ, Byrd DA, et al. (2007) Updated research nosology for HIV-associated neurocognitive disorders. Neurology 69 1789-1799.

4. Mind Exchange Working Group (2013) Assessment, diagnosis and treatment of HIV-associated neurocognitive disorder: A consensus report of the mind exchange program. Clin Infect Dis 57: 1004-1017.

5. Thames April D, Kim MS, Becker BW, Foley JM, Hines LJ, et al. (2011) Medication and finance management among HIV-infected adults: The impact of age and cognition. J Clin Exp Neuropsychol 33: 200-209.

6. Effros RB, Fletcher CV, Gebo K, Halter JB, Hazzard WR, et al. (2008) Workshop on HIV infection and aging: What are known and future research directions. Clin Infect Dis 47: 542-553.

7. Hasse B, Ledergerber B, Egger M, Vernazza P, Furrer H, et al. (2011) Aging and non-HIV-associated co-morbidity in HIV1 persons: The SHCS. 18th CROI Conference on Retroviruses and Opportunistic Infection, Boston, MA, USA.

8. Letendre S (2011) Central nervous system complications in HIV disease: HIVassociated neurocognitive disorder. Top Antivir Med 19: 137-142.

9. Gwen CL, Kandiah N, Chua A (2012) HIV-associated neurocognitive disorders (HAND) in a South Asian population-contextual application of the 2007 criteria. BMJ Open 2: e000662.

10. Eric GB, Burnam MA, Longshore D (2001) Psychiatric disorders and drug use among human immunodeficiency virus-infected adults in the United States. Arch Gen Psychiatry 58: 721-728.

11. Hemkens LG1, Bucher HC2 (2014) HIV infection and cardiovascular disease. Eur Heart J 35: 1373-1381.

12. Holt JL, Kraft-Terry SD, Chang $L$ (2012) Neuroimaging studies of the aging HIV-1-infected brain. J Neurovirol 18: 291-302

13. Morris Philip LP, Robinson RG, Raphael B (1993) Emotional lability after stroke. Austr N Z J Psychiatry 27: 601-605.

14. Lyketsos CG, Schwartz J, Fishman M, Treisman G (1997) AIDS mania. J Neuropsychiatry Clin Neurosci 9: 277-279.

15. Nakimuli-Mpungu E, Musisi S, Mpungu SK, Katabira E (2006) Primary mania versus HIV-related secondary mania in Uganda. Am J Psychiatry 163: 13491354.

16. Niemi M, Backman JT, Fromm MF, Neuvonen PJ, Kivistö KT (2003) Pharmacokinetic interactions with rifampicin: Clinical relevance. Clin Pharmacokinet 42: 819-850.

17. Rendic S, Di Carlo FJ (1997) Human cytochrome P450 enzymes: A status report summarizing their reactions, substrates, inducers and inhibitors. Drug Metab Rev 29: 413-580.
18. Rasmussen KG, Perry CL, Sutor B, Moore KM (2007) ECT in patients with intracranial masses. J Neuropsychiatry Clin Neurosci 19: 191-193.

19. Gardner DM, Murphy AL, O'Donnell H, Centorrino F, Baldessarini RJ (2010) International consensus study of antipsychotic dosing. Am J Psychiatry 167 686-693.

20. Marshman LA, Jakabek D, Hennessy M, Quirk F, Guazzo EP (2013) Posttraumatic amnesia. J Clin Neurosci 20: 1475-1481.

21. Decloedt EH, Maartens G (2013) Neuronal toxicity of efavirenz: A systematic review. Expert Opin Drug Saf 12: 841-846.

22. Ciccarelli N, Fabbiani M, Di Giambenedetto S, Fanti I, Baldonero E, et al. (2011) Efavirenz associated with cognitive disorders in otherwise asymptomatic HIVinfected patients. Neurology 76: 1403-1409.

23. Hartikainen S, Lönnroos E, Louhivuori K (2007) Medication as a risk factor for falls: Critical systematic review. J Gerontol 62: 1172-1181.

24. Newcomer JW (2007) Antipsychotic medications: metabolic and cardiovascular risk. J Clin Psychiatry 68: 8-13.

25. Lin K, Taylor MJ, Heaton R, Franklin D, Jernigan T (2011) Effects of traumatic brain injury on cognitive functioning and cerebral metabolites in HIV-infected individuals. J Clin Exp Neuropsychol 33: 326-334.

26. Kales HC, Valenstein M, Kim HM, McCarthy JF, Ganoczy D, et al. (2007) Mortality risk in patients with dementia treated with antipsychotics versus other psychiatric medications. Am J Psychiatry 164: 1568-1576.

27. de Vries OJ, Peeters G, Elders P, Sonnenberg C, Muller M, et al. (2013) The elimination half-life of benzodiazepines and fall risk: Two prospective observational studies. Age Ageing 42: 764-770.

28. Larson EB, Zollman FS (2010) The effect of sleep medications on cognitive recovery from traumatic brain injury. J Head Trauma Rehabil 25: 61-67.

29. Doyle KL, Morgan EE, Morris S, Smith DM, Little S, et al. (2013) Real-world impact of neurocognitive deficits in acute and early HIV infection. J Neurovirol 19: $565-573$

30. Cruess DG, Evans DL, Repetto MJ, Gettes D, Douglas SD, et al. (2003) Prevalence, diagnosis and pharmacological treatment of mood disorders in HIV disease. Biol Psychiatry 54: 307-316.

31. Vance DE, Mugavero M, Willig J, Raper JL, Saag MS (2011) Aging with HIV A cross-sectional study of comorbidity prevalence and clinical characteristics across decades of life. J Assoc Nurses AIDS Care 22: 17-25

32. APA Steering Committee on Practice Guidelines (2000) Psychiatry Online.

33. APA Guideline Watch (2006) Psychiatry Online.

34. Southern African HIV Clinician Society Guidelines (2013) $32 . \quad$ S o u the rn African HIV.

35. Cohen MA, Cozza KL, Bourgeois JA, Moghimi Y, Douaihy A (2016) The role of psychiatrists in HIV prevention. Psychiatr Times 30-32.

36. Cohen MA, Batista S (2010) A biopsychosocial approach to psychiatric consultation in persons with HIV and AIDS. Handbook of AIDS Psychiatry. Oxford University Press, New York 2010: 33-60.

37. Pyne JM, Fortney JC, Curran GM, Tripathi S, Atkinson JH, et al. (2011) Effectiveness of collaborative care for depression in human immunodeficiency virus clinics. Arch Intern Med 171: 23-31.

38. Heaton RK, Franklin DR, Deutsch R, et al. (2015) Neurocognitive change in the era of HIV combination antiretroviral therapy: The longitudinal CHARTER study. Infect Dis 60: 473-480.

39. Atkinson JH, Higgins JA, Vigil O, Dubrow R, Remien RH, et al. (2009) Psychiatric context of acute/early HIV infection. The NIMH multisite acute HIV infection study: IV. AIDS Behav 13: 1061-1067. 